\title{
Diagnóstico molecular de Mycobacterium tuberculosis en biopsias pleurales embebidas en parafina
}

\author{
Helí Barrón ${ }^{1,2}$, Mario Monteghirfo ${ }^{1}$, Nelson Rivera ${ }^{1,3}$
}

Resumen

Palabras clave
Objetivo: Determinar la sensibilidad de la prueba de reacción en cadena de la polimerasa(TBPCR) frente a la tinción de fluorocromo auramina (AFB-auramina) en muestras histológicas de biopsias pleurales embebidas en parafina, en el diagnóstico de tuberculosis pleural. Materiales y Métodos: Se usó 48 bloques de parafina obtenidos de los archivos de Patología del Hospital San José del Callao y del Instituto Nacional de Enfermedades Neoplásicas, 30 de los cuales tenían diagnóstico clínico de tuberculosis y 18 presentaron diagnóstico diferente de tuberculosis. Resultados: De los 30 casos con diagnóstico clínico de tuberculosis, 29 resultaron ser TB-PCR positivos. Los 18 casos negativos para tuberculosis resultaron también negativos para TBPCR. La sensibilidad y el valor predictivo negativo para TB-PCR fueron 96,7\% y 94,7\%. La sensibilidad y el valor predictivo negativo para AFB-auramina fueron 58,6\% y 56,7\%. En ambos casos, el valor predictivo positivo fue de 100\%. Conclusión: TB-PCR ha resultado ser un método muy sensible en el diagnóstico de tuberculosis en muestras histológicas embebidas en parafina.

Mycobacterium tuberculosis; tuberculosis pleural; biopsia; diagnóstico clínico.

\section{Molecular Diagnosis of Mycobacterium tuberculosis in paraffin-embedded pleural biopsies}

Abstract

Objective: To determine the sensitivity of the test tuberculosis polymerase chain reaction (TB-PCR) compared to fluorochrome auramine " $O$ " staining in histological embedded tissues of pleural biopsy in diagnosis of pleural tuberculosis. Materials and Methods: We used 48 formalin-fixed paraffin-embedded tissue obtained from the archives of the Department of Pathology, Hospital San Jose del Callao, and Instituto Nacional de Enfermedades Neoplasicas. Thirty cases had clinical diagnosis of tuberculosis and 18 different diagnosis. Results: Twenty-nine of 30 cases having tuberculosis were TB-PCR positive. All 18 negative cases were TB-PCR negative. The sensibility and the negative predictive value of TBPCR were $96,7 \%$ and 94,7\% respectively. On the other hand, the sensitivity and negative predictive value of AFB-Auramine were $58,6 \%$ and $56,7 \%$. The positive predictive value was $100 \%$ in both cases. Conclusion: TB-PCR is a very sensitive method for TBdiagnosis in formalin-fixed, paraffin embedded pleural biopsies.

1 Centro de Investigación de Bioquímica y Nutrición Alberto Guzmán Barrón. Facultad de Medicina, UNMSM. Lima, Perú.

2 Hospital San Jose, Minsa. Callao, Perú.

3 Morgue Central. Lima, Perú.
Keywords: Mycobacterium tuberculosis; tuberculosis, pleural; biopsy; diagnosis, clinical.

\section{INTRODUCCIÓN}

La tuberculosis, aunque curable, sigue siendo una de las principales causas de muerte por agentes infecciosos en el mundo. Ha sido declarada por la Organización Mundial de la Salud (OMS) como un problema de emergencia global, debido principalmente al sinergismo entre el bacilo tuberculoso y el virus de la inmunodeficiencia humana (VIH). La magnitud de este problema se ve reflejado por la aparición de nuevos casos, que según datos de la OMS son de 10 millones en el 2002 y casi 12 millones de nuevos casos proyectados al $2005\left(^{(1,2,3,4)}\right.$.

El Mycobacterium tuberculosis es frecuentemente adquirido en etapas 
tempranas de la vida, como una infección aguda y con desarrollo de inmunidad, formación de granuloma y calcificación. Después, sigue un largo periodo de latencia, que puede reactivarse en algunos individuos.

El Mycobacterium tuberculosis o bacilo de Koch es el agente etiológico de la tuberculosis. Se trata de un bacilo aerobio estricto, ácido-alcohol resistente, no tiene movilidad, es de crecimiento lento; esta bacteria se inactiva con radiación ultravioleta y/o temperaturas por encima de $60^{\circ} \mathrm{C}$. Varias especies similares integran el complejo $M$. tuberculosis: $M$. bovis, $M$. africanum y $M$. microti. El bacilo BCG (M. bovis atenuado) también puede ocasionar una enfermedad en pacientes inmunocomprometidos, la que no se puede distinguir clínicamente de la tuberculosis. La infección se da principalmente a través de secreciones respiratorias que son expulsadas del paciente en el momento de la tos. En el periodo de incubación, que puede variar desde el momento de la infección hasta 90 días, la bacteria crece lentamente en los macrófagos, pudiendo diseminarse a otras partes del cuerpo del hospedero. En la mayoría de casos, esta enfermedad es controlada por la inmunidad celular, de los cuales un $10 \%$ desarrollará la enfermedad activa a lo largo de su vida $\left({ }^{5}\right)$.

Los últimos datos de la OMS, que datan del año 2003, indican una prevalencia de 15,4 millones de infectados, lo que representa una tasa de 245/100,000 habitantes. Esto representa 8,8 millones de casos de nuevos de tuberculosis cada año. La mortalidad actual de esta enfermedad es de 2 millones de muertes anuales, de las cuales $98 \%$ ocurren en países subdesarrollados. Esta situación podría agudizarse más debido a que las pruebas convencionales de diagnóstico de TB demoran 8 semanas, ocasionando un retraso en el tratamiento, por lo que la rápida identificación es esencial para el manejo del paciente $\left(^{6}\right)$.
En el Perú, en el año 2004 se registró 64 276 pacientes con diagnóstico de tuberculosis y la prevalencia de TB en nuestro país va en aumento. La tasa de incidencia es de 66,39 por cada 100000 habitantes, comparable con Bolivia, Ecuador, Guyana y Haití $\left({ }^{7}\right)$.

El diagnóstico de la TB depende de la historia clínica del paciente, exámenes bacteriológicos, pruebas de rayos $\mathrm{X}$, prueba de tuberculina, entre otros. El diagnóstico etiológico de TB depende de la demostración microscópica de los bacilos alcohol ácido resistentes (BAAR) y de su aislamiento e identificación en medios de cultivo $\left({ }^{8}\right)$.

A pesar de los grandes avances en los métodos de diagnóstico, la tuberculosis es todavía identificada por los antiguos procedimientos, tales como la tinción de Ziehl-Neelsen y por el cultivo en el medio de Lowestein-Jensen, que todavía son considerados como estándar de oro en el diagnóstico de TB, debido a que los procedimientos modernos son aún muy costosos $\left({ }^{8,9}\right)$.

Aunque la baciloscopia o tinción BAAR en esputo es un método de diagnóstico simple y relativamente rápido, presenta problemas en cuanto a su sensibilidad $(50 \%)$, ya que son necesarias cantidades mayores de 10000 bacilos / $\mathrm{mL}$ para su detección; así, casi la mitad de los casos de TB activa puede tener baciloscopia negativa. Esta sensibilidad puede estar aún más disminuida en los casos de biopsias embebidas en parafina.

Las pruebas de cultivo de esputo son más sensibles ( 80 a $96 \%)$, sensibilidad que disminuye si se trata de muestras con menor carga bacilar. tales como liquido cefalorraquídeo, orina o biopsias. El principal inconveniente de este método es el tiempo de incubación para la recuperación de este microorganismo, que puede demorar hasta 8 semanas. 
Actualmente, las técnicas moleculares permiten la identificación de micobacterias a nivel de especie en aquellas muestras donde los métodos de cultivo y otras técnicas convencionales de detección son negativas.

Últimamente, se está haciendo extensivo el uso de métodos de amplificación de genes, tanto para la identificación del bacilo tuberculoso a partir de cultivos y muestras clínicas como para la detección molecular de resistencia a drogas. Así, tenemos la técnica de la PCR con gran sensibilidad, que en condiciones óptimas puede detectar de 1 a 10 microorganismos. Con los métodos de extracción de DNA disponibles, es posible ahora la identificación de micobacterias a partir de cualquier muestra biológica $\left({ }^{10}\right)$.

La técnica de PCR IS6110 es la que presenta mayor sensibilidad y especificidad para el diagnóstico de Mycobacterium tuberculosis $\left({ }^{11,12}\right)$.

Actualmente, se usa muchos genes y secuencias para su amplificación. Así, tenemos secuencias repetitivas $y$ no repetitivas. IS6110 de $123 \mathrm{pb}$ es una secuencia repetitiva frecuentemente usada; en tanto que otra secuencia, la rRNA16S de $306 \mathrm{pb}$, es una secuencia no repetitiva $\left({ }^{13}\right)$.

IS6110 es un elemento genético de inserción que genéricamente se le denomina IS, del inglés insertion sequence; ésta representa secuencias de ADN dispersas en los genomas bacterianos con actividad de transposición. La secuencia IS6110 solo es detectable en genomas bacterianos del complejo Mycobacterium tuberculosis y tiene la estabilidad necesaria para ser utilizada como herramienta de diagnóstico $\left({ }^{14}\right)$.

El objetivo de este estudio fue evaluar la sensibilidad de la prueba de reacción en cadena de la polimerasa (TB-PCR) frente a la tinción de fluorocromo auramina (AFBauramina) en muestras histológicas de biopsias pleurales embebidas en parafina en el diagnóstico clínico de tuberculosis pleural.

\section{MATERIALES Y MÉTODOS}

El estudio fue de tipo observacional y transversal.

Los especímenes que fueron incluidos para este trabajo estaban conformados por 30 muestras de biopsias pleurales fijadas en formalina y embebidas en parafina, diagnosticadas histológicamente como pleuritis crónica, pleuritis tuberculoide, reacción granulomatosa. Los especímenes fueron obtenidos de los archivos del Servicio de Anatomía Patológica del Hospital San José del Callao (HSJ) y correspondían a muestras procesadas durante el año 2004. Además, se incluyó en este estudio 18 muestras de biopsias pleurales con diagnóstico negativo de TB pleural o algún otro tipo de lesión que sugería diagnóstico de TB, todas procedentes del Instituto Nacional de Enfermedades Neoplásicas (INEN).

Para la tinción de auramina ' $\mathrm{O}$ ', se usó cortes de 5 ìm de espesor y la tinción de realizó siguiendo el procedimiento clásico de Ziehl Neelsen. Este es el método más rápido para confirmar la enfermedad micobacteriana. La muestra se tiñe con carbolfucsina (colorantes de Ziehl-Neelsen y Kinyoun) o con fluorocromos (como auramina), luego se decolora con solución acido-alcohol y luego es sometido a contra tinción. Finalmente, es examinado con el microscopio óptico o con el de fluorescencia (en caso de fluorocromos). La tinción con fluorocromos es más sensible, puesto que la fluorescencia puede ser detectada rápidamente a bajo aumento, y después se confirma empleando un aumento mayor. 
El aislamiento de ADN fue realizado a partir de cortes histológicos de 20 um de las muestras embebidas en parafina. El desparafinado se hizo con xilol y etanol. La extracción de ADN estuvo basada en cloroformo-proteinasa $\mathrm{K}$.

Para la amplificación de las secuencias de insercion IS6110, se utilizó $5 \mathrm{uL}$ de las muestras de ADN de Mycobacterium obtenidos en el paso anterior. La amplificación PCR IS6110 fue realizada según el método descrito por Park y col. $\left({ }^{4}\right)$. Los productos de PCR IS6110 fueron analizados mediante electroforesis en agarosa al $2 \%$. La tinción de los geles se realizó con bromuro de etidio. Se visualizó las bandas de $123 \mathrm{pb}$ empleando un transiluminador UV.

Se evaluó la especificidad y la sensibilidad de los métodos comparados con los criterios estándares de diagnóstico clínico de TB. Asimismo, se obtuvo el valor predictivo positivo (VPP) y el valor predictivo negativo (VPN) para cada uno de los métodos de diagnóstico $\left({ }^{15,16}\right)$.

\section{RESULTADOS}

De 1 los 48 casos estudiados, 30 fueron clínicamente diagnosticados como tuber-

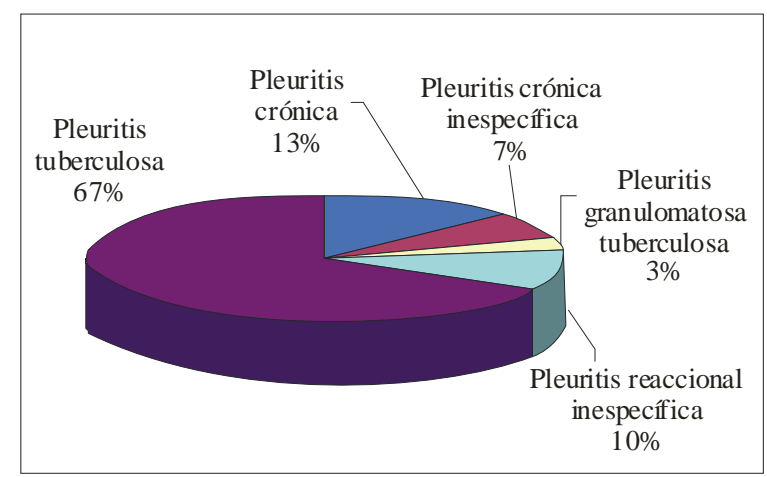

Figura 1. Diagnóstico histopatológico de los casos clínicamente diagnosticados como tuberculosis pleural. culosis pleural, de acuerdo a los datos obtenidos de la revisión de las historias clínicas del año 2004. De las 30 muestras diagnosticadas como TB, se encontró que $20(67 \%)$ correspondieron a diagnóstico histopatológico de pleuritis tuberculosis y, siguiendo el orden de frecuencia, 4 casos (13\%) de pleuritis crónica, 3 casos (10\%) presentaron pleuritis reaccional inespecífica, 2 pacientes (7\%) pleuritis crónica inespecífica y un paciente $(3 \%)$ pleuritis granulomatosa tuberculosa. Figura 1.

Los 18 casos restantes tuvieron diagnóstico clínico e histológico diferente de TB, de acuerdo a los datos de revisión de historia clínica de los archivos del INEN del año 2004.

De los 20 casos que fueron diagnosticados histológicamente como pleuritis tuberculosa, se encontró que $16(80 \%)$ fueron positivos para la tinción AFB-auramina, y todas 20 $(100 \%)$ fueron positivas para IS6110 TBPCR (Tabla 1 y Figura 2).

Se encontró que de los 4 casos que tenían diagnóstico histopatológico de pleuritis crónica, ninguno $(0 \%)$ fue positivo para la prueba de AFB-auramina, en tanto que todas fueron positivas para IS6110 TB-PCR (Tabla 2). Del mismo modo, entre los 3 casos diagnosticados como pleuritis reaccional inespecífica, ninguna fue positiva para AFB-auramina y todas presentaron positividad para IS6110 TB-PCR (ver Tabla $1)$.

Entre los 2 casos de pleuritis crónica inespecífica, solo $1(50 \%)$ fue AFBauramina positiva, en tanto que los 2 casos fueron IS6110 TB-PCR positivas, tal como se muestra en la Tabla 1.

Finalmente, el único caso de pleuritis granulomatosa tuberculosa presentó diagnóstico negativo para AFB-auramina y diagnóstico positivo para IS6110 TBPCR. 
Diagnóstico molecular de Mycobacterium tuberculosis

Tabla 1. Comparación de casos por diagnóstico histopatológico, tinción de auramina y resultados de TB PCR.

\begin{tabular}{|c|c|c|c|c|c|c|}
\hline \multirow[b]{2}{*}{ Diagnóstico histopatológico } & \multicolumn{3}{|c|}{ Tinción de auramina } & \multicolumn{3}{|c|}{ TB-PCR } \\
\hline & Negativo & Positivo & & Negativo & Positivo & \\
\hline Pleuritis crónica & 4 & 0 & 4 & 1 & 3 & 4 \\
\hline Pleuritis crónica inespecífica & 1 & 1 & 2 & 0 & 2 & 2 \\
\hline Pleuritis granulomatosa tuberculosa & 1 & 0 & 1 & 0 & 1 & 1 \\
\hline Pleuritis reaccional inespecífica & 3 & 0 & 3 & 0 & 3 & 3 \\
\hline Pleuritis tuberculosa & 4 & 16 & 20 & 0 & 20 & 20 \\
\hline \multirow[t]{2}{*}{ Diagnóstico negativo de TB } & 18 & 0 & 18 & 18 & 0 & 18 \\
\hline & 31 & 17 & 48 & 19 & 29 & 48 \\
\hline
\end{tabular}

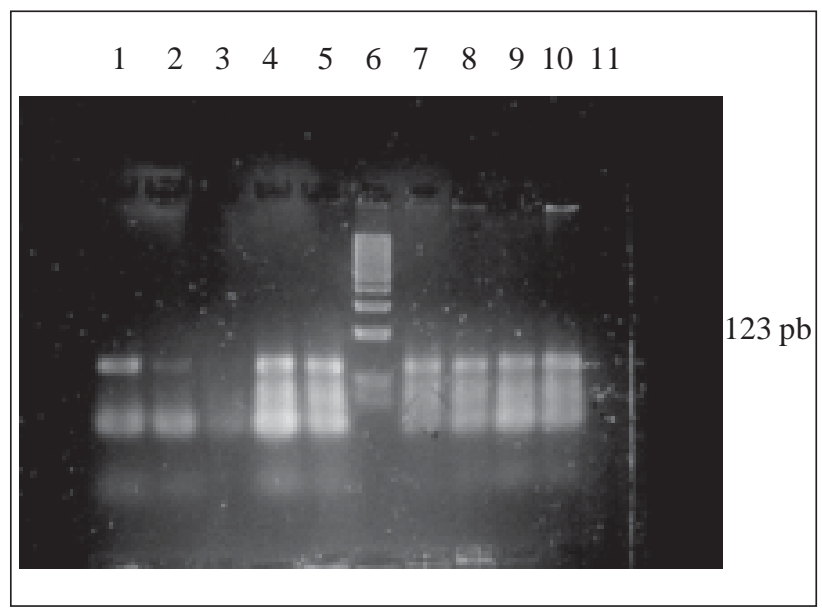

Figura 2. Los carriles 1, 2, 5, 7, 8, 9 ,10 presentan resultados positivos de 123 pares de bases. Carril 3 con resultado negativo. Carril 4 control positivo a partir de cultivo. Carril 6 marcador de tamaño molecular de 100 pb. Carril 11 control negativo.

Entre los resultados del diagnóstico clínico y los resultados de la prueba de IS6110 TB-PCR se encontró una sensibilidad de $96,7 \%$, una especificidad de $100 \%$, VPN de $94,7 \%$ y VPP de $100 \%$ para la prueba de IS 6110 TB-PCR.

Así mismo, entre los resultados del diagnóstico clínico y los resultados de la prueba de AFB-auramina, se encontró una sensibilidad de $58,6 \%$, una especificidad de $100 \%$, VPN de $56,7 \%$ y VPP de $100 \%$.
Se observó una marcada diferencia $(40 \%)$ entre los resultados comparativos de las sensibilidad de las pruebas IS6110 TB PCR y AFB-auramina, así como, una diferencia de casi $40 \%$ entre los VPN de ambas pruebas en estudio.

\section{DISCUSIÓN}

Las estadísticas presentadas por la OMS revelan que un tercio de la población mundial está infectada con tuberculosis, resultando en 8 a 10 millones de nuevos casos de tuberculosis activa cada año, lo cual significa un gran desembolso de dinero de parte de los gobiernos y sus programas de salud en el diagnóstico y tratamiento de esta enfermedad $\left({ }^{1,2,6,7}\right)$.

Los métodos de diagnóstico bacteriológicos tradicionales se basan principalmente en el método de tinción de Ziehl-Neelsen y el cultivo para el aisla-

Tabla 2. Comparación entre los resultados de TB PCR y los resultados de la tinción de auramina.

\begin{tabular}{lccc}
\hline & \multicolumn{3}{c}{ Tinción de auramina } \\
\cline { 2 - 4 } TB PCR & Positivo & Negativo & Total \\
\hline \multirow{2}{*}{ Positivo } & 17 & 12 & 29 \\
Negativo & 0 & 19 & 19 \\
& 17 & 31 & 48 \\
\hline
\end{tabular}


miento e identificación de Mycobacterium tuberculosis. La técnica de Ziehl-Neelsen, que presenta el mismo principio que la tinción de auramina, aunque es rápida y muy barata, tiene muy baja sensibilidad, especialmente en muestras histológicas embebidas en parafina. El cultivo, que es bastante sensible ya que se requiere solamente la presencia de 10 a 100 microorganismos viables por muestra, es un procedimiento relativamente largo que puede tomar de 4 a 8 semanas para completarse. Además, el cultivo puede presentar una sensibilidad menor en muestras histológicas, menor aún cuando el paciente ha sido tratado parcialmente, ya que se requiere de la presencia de microorganismos viables para su aislamiento en los medios de cultivo $\left({ }^{1,2,3,4,17}\right)$.

En los últimos años, han aparecido pruebas moleculares que superan largamente la sensibilidad y la especifidad de las pruebas tradicionales para el diagnóstico de tuberculosis a partir de especimenes histológicos. Entre estos métodos se incluye la técnica de PCR y otras técnicas de amplificación que detectan ADN de Mycobacterium tuberculosis $\left({ }^{4,11,18}\right)$.

Diversos reportes muestran la utilidad de la técnica de PCR como método diagnóstico rápido de tuberculosis en diversos tejidos $(4,11,18,19,20,21)$; en nuestro caso, todos los especímenes estudiados corresponden a biopsias pleurales con diversos diagnósticos histológicos.

En este estudio, se analizó 48 muestras de biopsias pleurales de pacientes fijadas en formalina y embebidas en parafina, 30 de las cuales tenían diagnóstico clínico de tuberculosis. También, se analizó 18 muestras de biopsias pleurales de pacientes que presentaron diagnóstico clínico e histológico diferente de tuberculosis.

No se usó el cultivo de Mycobacterium tuberculosis como referencia para TB-PCR; tampoco se usó este criterio para la tinción AFB-auramina, debido que las biopsias pleurales no fueron enviadas para cultivo. En contraste, se empleó el diagnóstico clínico como referencia comparativa tanto para TB-PCR como para la tinción AFBauramina.

Se encontró que la sensibilidad de la técnica de TB-PCR fue de $96,6 \%$ y una especificidad de $100 \%$, en tanto que el valor predictivo positivo fue de $100 \%$ y el valor predictivo negativo de $94,7 \%$, para especímenes histológicos provenientes de biopsias pleurales.

Un estudio publicado por Park y col. $\left({ }^{4}\right)$, en Corea, mostró una sensibilidad de $78 \%$ y una especificidad de $88 \%$ para TB-PCR; además, Park encontró un valor predictivo positivo de $93 \%$ y un valor predictivo negativo de $66 \%$. Estos resultados difieren de los obtenidos en este estudio, lo que puede explicarse por la variedad de muestras empleadas en el ensayo de Park, quien incluyó muestras de diversos sitios anatómicos, tales como pulmón, pleura, tejidos blandos y tejido gastrointestinal, incluyendo hígado, estómago y duodeno, entre otros, que tenían sospecha clínica de tuberculosis; en tanto que, en nuestra investigación todos los especímenes provenían de biopsias pleurales.

Por otro lado, los resultados de sensibilidad y especificidad de la prueba TB-PCR encontrados en el presente trabajo son muy similares a los obtenidos por Isik Somuncu y col., quienes obtuvieron una sensibilidad y especificidad de $90 \%$ y $100 \%$, respectivamente $\left({ }^{5}\right)$. Cabe mencionar que el estudio de Isik Somuncu se basó en tejidos que presentaban infección granulomatosa necrotizante.

En una investigación publicada el año $2003\left({ }^{11}\right)$, Fukunaga y col. realizaron un estudio comparativo entre la técnica de tinción de BAAR y PCR en tiempo real, 
para el cual emplearon 30 muestras embebidas en parafina que tenían diagnóstico de lesión granulomatosa. En ese estudio, se demostró que el tratamiento con formalina y con xilol, que son reactivos empleados en el procesamiento de muestras histológicas, puede disminuir drásticamente la sensibilidad de las técnicas de tinción para micobacterias, tales como ZiehlNeelsen y la tinción de auramina-rodamina. Todo esto explicaría la baja sensibilidad para la prueba AFB-auramina en muestras embebidas en parafina, como la que se ha encontrado en nuestra investigación, que presenta $40 \%$ menor sensibilidad que TBPCR.

Punkae y col. (13), en Tailandia, 2005, encontraron una sensibilidad de $29,3 \%$ y especificidad de $61,1 \%$. valores bastante bajos para PCR en biopsias embebidas en parafina. Estos resultados difieren grandemente con los resultados obtenidos en el presente estudio. La explicación de esta diferencia es que Punkae emplea solamente biopsias de piel. Además, Punkae empleó un método de PCR basado en $16 \mathrm{~S}$ rARN, en contraste a nuestro estudio que ha empleado la secuencia IS 6110 .

Finalmente, para la confirmación de estos diagnósticos es necesario realizar las pruebas de tinción BAAR y el cultivo, pero se sabe que los procedimientos de tinción para BAAR tales como Ziehl-Neelsen y AFB-auramina presentan baja sensibilidad en muestras histológicas y además no existe disponibilidad para procesar dichos cultivos. Por lo tanto, se debe considerar seriamente el empleo de técnicas de biología molecular como métodos rápidos de ayuda diagnóstica. Sin embargo, dado que este es un procedimiento de diagnóstico todavía laborioso en nuestro medio, se debe seguir estrictos controles para evitar contaminaciones cruzadas y / o falsos positivos.

\section{A GRADECIMIENTOS}

A la Dra. Raquel Oré Sifuentes, jefe de la Maestría en Bioquímica UNMSM, al Dr. Pedro Pablo Díaz Velásquez del Hospital San José y al Dr. Carlos Barrionuevo del Instituto Nacional de Enfermedades Neoplásicas.

\section{REFERENCIAS BIBLIOGRÁFICAS}

1. Buck GE, O'Hara LC, Summersgill JT. Rapid, simple method for treating clinical specimens containing Mycobacterium tuberculosis to remove DNA for polymerase chain reaction. J Clin Microbiol. 1992;30:1331-4.

2. Jimenez D, Diaz G, Perez-Rodriguez E. Diagnosis of pleural tuberculosis. Chest. 2001:121(3):1005.

3. Young DB. Mycobacteria research in the post-genomic era. Microbiology. 2002;148:2915-7.

4. Park DY, Kim JY, Choi KU, Lee JS, Lee CH, Sol MY, et al. Comparison of polymerase chain reaction with histopathologic features for diagnosis of tuberculosis in formalin-fixed paraffin-embedded histologic specimens. Arch Pathol Lab Med. 2003;127:326-30.

5. Parrish NM, Dick JD, Bishai WR. Mechanisms of latency in Mycobacterium tuberculosis [review]. Trends Microbiol. 1998;6(3):107-12.

6. Organización Panamericana de la Salud, Programa Regional de Tuberculosis. Tuberculosis. 2003;6(1):1-6.

7. Situación Epidemiológica de la TB. (Región de las Ameritas, 2004). En http://www.paho.org/spanish/ad/dpc/cd/1

8. Sociedad Argentina de Pediatría, Comité Nacional de Neumología, Comité Nacional de Infectología. Criterios de diagnóstico y tratamiento de la tuberculosis infantil. Arch Argent Pediat. 2002;100(2).

9. Katoch VM. Newer diagnostic techniques for tuberculosis. Review Article. Indian J Med Res. 2004;120:418-28.

10. Hsiao P-F, Tzen C-Y, Chen H-C, Su H-Y. Polymerase chain reaction based detection of Mycobacterium tuberculosis in tissues showing granulomatous inflammation without demonstrable acid-fast bacilli. International Journal of Dermatology. 2003;42:281-6.

11. Johansen IS, Thomsen VO, Forsgren A, Hansen BF, Lundgren B. Detection of Mycobacterium tuberculosis complex in formalin-fixed, paraffin-embedded tissue specimens with necrotizing granulomatous inflammation by strand displacement amplification. J Mol Diagn. 2004;6:2315 .

12. Lodha R, Kabra SK. Newer diagnostic modalities for tuberculosis. The Indian Journal of Pediatrics. 2004;71(3):221-7.

13. Mahaisavariya P, Chaiprasert A, Manonukul J, Khemngern S, Tingtoy N. Detection and identification of Mycobacterium species by polymerase chain reaction (PCR) from paraffinembedded tissue compare to AFB staining in pathological sections. J Med Assoc Thai. 2005;88(1):108-13. 
14. Fomukong NG, Tang TH, al-Maamary S, Ibrahim WA, Ramayah S, Yates M, et al. Insertion sequence typing of Mycobacterium tuberculosis: characterization of a widespread subtype with a single copy of IS6110. Tuber Lung Dis. 1994;75(6):435-40.

15. Altman DG, Bland JM. Statistics notes: Diagnostic tests 1: sensitivity and specificity. BMJ. 1994;308:1552.

16. Altman DG, Bland JM. Statistics notes: Diagnostic tests 2: predictive values. BMJ. 1994;309:102.

17. Nagesh BS, Sehgal S, Jindal SK, Arora SK. Evaluation of polymerase chain reaction for detection of Mycobacterium tuberculosis in pleural fluid. Chest. 2001;119(6):1737-41.

18. Zink AR, Nerlich AG. Molecular strain identification of the Mycobacterium tuberculosis complex in archival tissue samples. J Clin Pathol. 2004;57:1185-92.

19. Niemann S, Richter E, Rusch-Gerdes S. Stability of Mycobacterium tuberculosis IS6110 restriction fragment length polymorphism patterns and spoligotypes determined by analyzing serial isolates from patients with drug-resistant tuberculosis. J Clin Microbiol. 1999;37(2):409-12.

20. Cho MS, Lee SN, Sung SH, Han WS. Comparison of ZiehlNeelsen stain and TB-PCR on detection of Mycobacterium tuberculosis in formalin-fixed, paraffin-embedded tissues of chronic granulomatous inflammation [abstract]. The Korean Journal of Pathology. 2003;37:379-83.

21. Osman C, Karsligil T, Bayazit N. Evaluation of a polymerase chain reaction amplification method for Mycobacterium tuberculosis complex on samples from different sources. Scand J Infect Dis. 2003;35:329-31.

Manuscrito recibido el 15 de febrero de 2006 y aceptado para publicación el 10 marzo de 2006.

\section{Correspondencia: Helí J. Barrón Pastor}

Centro de Investigación de Bioquímica y Nutrición Facultad de Medicina, UNMSM.

Av. Grau 750. Lima 1, Perú

Correo-e: helibarron@unmsm.edu.pe 